\title{
Virgin domain structures in mesoscopic Co patterns: Comparison between simulation and experiment
}

\author{
S. Cherifi \\ Laboratoire Louis Néel, Centre National de la Recherche Scientifique (CNRS), BP 166, 38042 Grenoble \\ Cedex 9, France \\ R. Hertel ${ }^{\mathrm{a})}$ and J. Kirschner \\ Max-Planck-Institut für Mikrostrukturphysik, Weinberg 2, 06120 Halle, Germany
}

H. Wang

Arizona State University, Tempe, Arizona 85287

R. Belkhou

Laboratoire pour l'Utilisation du Rayonnement Electromagnétique (LURE), Bat 209D, Université Paris-Sud, B.P. 34, 91898 Orsay Cedex, France

A. Locatelli and S. Heun ${ }^{\text {b) }}$

Sincrotrone Trieste, S.S. 14, km 163.5, 34012 Basovizza (Trieste), Italy

A. Pavlovska and E. Bauer ${ }^{\mathrm{c}}$

Arizona State University, Tempe, Arizona 85287 and Sincrotrone Trieste, S.S. 14, km 163.5, 34012 Basovizza (Trieste), Italy

(Received 14 October 2004; accepted 1 July 2005; published online 16 August 2005)

\begin{abstract}
The magnetization states of 20-nm-thick rectangular Co thin-film elements are studied with micromagnetic modeling and $\mathrm{x}$-ray magnetic circular dichroism photoemission electron microscopy. The energies of ten domain configurations obtained in the modeling are compared with the frequency of occurrence of the corresponding virgin domain structures as a function of aspect ratio from 1:1 to $1: 3$ and of width from 200 to $600 \mathrm{~nm}$. The results show that the abundance of the virgin states is largely determined by the magnetic energy densities of the elements. (C) 2005 American Institute of Physics. [DOI: 10.1063/1.2007872]
\end{abstract}

\section{INTRODUCTION}

The memory elements in magnetic storage devices are today in the 100-nm range. Their switching behavior and remanent state depend on the relative energies of the various domain configurations. Therefore, a theoretical understanding of these energies and its verification by experiment is of fundamental importance. For this reason, numerous studies have already been made in the past (see, for example, Refs. 1 and 2). In the simulations the size of the elements as well as the accuracy were limited by computer power and in the experiments the size of the elements by the resolution of the magnetic imaging methods. The rapid improvements in computer memory and processing time and the developments in magnetic imaging in recent years allow now a systematic study of a large number of elements of identical size, shape, and thickness by simulation and experiment.

On the simulation side, Hertel $^{3}$ has developed computational schemes that allow accurate calculations of the magnetic structure of micron-sized soft-magnetic materials. Recently ${ }^{4}$ he has applied these methods to calculate the energies of five magnetization structures in rectangular permal-

\footnotetext{
${ }^{\text {a) }}$ Present address: Institute of Solid State Research (IFF), Research Center Jülich, 52425 Jülich, Germany.

${ }^{b)}$ Present address: TASC-INFM Laboratory, S.S. 14, km 163.5, 34012 Basovizza (TS), Italy.

${ }^{c}$ Author to whom correspondence should be addressed; electronic mail: ernst.bauer@asu.edu
}

loy films with the aspect ratio $2: 1$ that allowed him to develop a phase diagram of the lowest energy configurations as a function of thickness and length. He found that in the thinnest films quasihomogeneous states, the so-called $C$ and $S$ states, had the lowest energy and that with increasing thickness and length flux-closure patterns, the so-called Landau and later the diamond pattern were the most stable configurations.

On the experimental side, $\mathrm{x}$-ray magnetic circular dichroism photoemission electron microscopy (XMCDPEEM), as studied by Stoehr et al., ${ }^{5}$ has reached now a resolution in the $10-\mathrm{nm}$ range ${ }^{6}$ so that the domain structure in elements in the $100-\mathrm{nm}$ range can be imaged. Thus large-scale simulation and magnetic imaging can be done on the same size scale, allowing a direct comparison between theory and experiment. In this paper we present the results of an XMCDPEEM study of 20-nm-thick rectangular Co elements with aspect ratios of 1:1, 1:2, and 1:3 and widths of 200, 400, and $600 \mathrm{~nm}$ and compare them with micromagnetic simulations using the computational procedures mentioned above. ${ }^{3,4}$ If we assume that the probability of a domain structure is determined by its total energy $E$ and not by accidental domain nucleation, then there should be a close correlation between simulation and experiment. This will only be true for the virgin state because once the element has been magnetized, it may not be able to return from the magnetized state to the state of lowest energy due to insurmountable potential barri- 
ers. It may end up in a metastable remanent state with intermediate energy or even remain in its magnetized state. For this reason only virgin samples are considered in this work.

\section{EXPERIMENTAL PROCEDURES}

The samples were prepared on a native oxide-covered $\mathrm{Si}$ wafer by spin coating with polymethyl-methacrylate (PMMA) photoresist, patterning it by electron-beam lithography (EBL) in a JBX-6000FS/E EBL instrument and developing it. After development of the PMMA a 10-nm Al, 20-nm Co, and a 2.5-nm-thick corrosion-inhibiting Al layer were deposited in sequence via electron-beam evaporation at a rate of $0.04 \mathrm{~nm} / \mathrm{s}$. The final lift-off processing removes all deposited material except the rectangular pattern. The elements in the patterns were separated sufficiently so that there was negligible magnetic coupling via their stray fields. In order to have enough statistics many elements of a given size and shape were EBL written, ranging from 100 for the smallest (200-nm width and 1:1 aspect ratio) to 18 for the largest (600-nm width and 1:3 aspect ratio).

After transferring to the XMCDPEEM instrument the samples were first sputtered to reduce the thickness of the top Al layer and to eliminate remnants of the photoresist before introducing them into the main chamber of the instrument. The instrument was the commercial spectroscopic photoemission and low-energy electron microscope (SPELEEM) (Ref. 7) operating at the nanospectroscopy beamline of the synchrotron radiation facility ELETTRA. ${ }^{8}$ This beamline is served by an undulator that produces the circular polarized light in the energy range needed for these XMCD measurements. The light is monochromatized and focused into a 25 $\times 5-\mu \mathrm{m}^{2}$ (in the horizontal and vertical directions, respectively) microspot on the sample. The spot is horizontally elongated due to grazing incidence. In the energy range of Co $2 p_{3 / 2}\left(L_{3}\right)$ the photon flux into this spot is 6 $\times 10^{10}$ photons/s, which allows acquisition of images in the range from 1 to $5 \mathrm{~min}$, for images with a field of view of $5-\mu \mathrm{m}$ diameter. Two images with opposite helicity were taken and subtracted to produce the magnetization distribution images shown in Figs. 3-5. The contrast in the images is proportional to $\boldsymbol{\sigma} \cdot \mathbf{M}$, where the axial vector $\boldsymbol{\sigma}$ gives the direction of the incident light and its helicity and $\mathbf{M}$ is the local magnetization. LEEM and mirror electron microscopy (MEM) images were taken too in order to obtain information on a preferred crystallite orientation and grain size.

\section{MICROMAGNETIC MODELING}

Micromagnetic simulations have been employed to model the magnetic structure in rectangular Co thin-film elements of different sizes and shapes. The simulations yielded a large "zoo" of possible stable magnetization states. Even if the sample size, shape, and material are identical, completely different equilibrium, zero-field magnetization states may result, depending on the initial conditions of the simulation. The variety of possible magnetization states reflects the phenomenon of hysteresis and metastability, which is ubiquitous in ferromagnetism. The simulations were performed with a micromagnetic code based on finite elements that has been developed by one of us. ${ }^{3}$ The sample volume was subdivided into tetrahedral finite elements and the directional field of the magnetization was discretized at the corner points of each tetrahedron. The stray field was calculated by solving Poisson's equation for the magnetic scalar potential $U$, from which the stray field was derived as a gradient field. The exact consideration of the boundary conditions for $U$ was achieved with a combination of the finite element method and the boundary element method. Equilibrium magnetic structures were obtained by means of energy minimization using the conjugate gradient method. A more detailed description of the method is given in Refs. 3 and 4. Starting from a given magnetic structure, the program finds the nearest local minimum in the complex potential landscape in the magnetic configuration space. Therefore, by choosing different initial conditions for the magnetization distribution, one generally finds different converged states. A transition between two local minima, i.e., a conversion from one magnetization state into another, is hampered by an energetic barrier of unknown height. In a real sample, such a barrier can be overcome, usually on a very long time scale, by means of thermal excitations. These effects are not considered in the simulation.

In order to obtain numerous different magnetization states and compare their energies, the magnetic structure of each sample has been calculated several times, always with a different initial configuration. The initial configurations were chosen to coarsely resemble the expected final state, e.g., by placing a magnetic vortex on different positions in the rectangle and/or aligning the magnetization in different directions at the sample's edges, thus putting the system in the vicinity of an expected energy minimum. In many cases, the initial configuration was unstable and the particle dropped into a completely different magnetization state which was usually simpler than the one we aimed at. The starting configurations were deliberately chosen in a coarse way, bearing only weak resemblance to a real magnetization pattern, in order to ensure that the code performs several iterations to find a self-consistent solution, thus ruling out that the minimization finishes prematurely due to a "too good" initial guess.

The simulations provide information about the energy of a magnetization state, which is a quantity that is not accessible by the experiment.

\section{SIMULATION RESULTS}

Before presenting the magnetic domain structures observed in the experiment it is useful to look at the results of the simulations of these patterns. The simulations were made with the methods described in detail in Ref. 4, using the following parameters for Co: exchange constant $A=3.3$ $\times 10^{-11} \mathrm{~J} / \mathrm{m}$, zero uniaxial anisotropy constant $K_{u}$, and stray field energy constant $K_{d}=\mu_{0} M_{s}^{2} / 2=1.27 \times 10^{6} \mathrm{~J} / \mathrm{m}^{3}$ corresponding to a saturation magnetization $M_{s}=1.42 \times 10^{6} \AA / \mathrm{m}$. Although the magnetocrystalline anisotropy constant of crystalline Co is $K_{u}=5 \times 10^{5} \mathrm{~J} / \mathrm{m}^{3}$, the random orientation of the exchange-coupled, nanosized grains in our samples effectively makes the material amorphous from the magnetic 

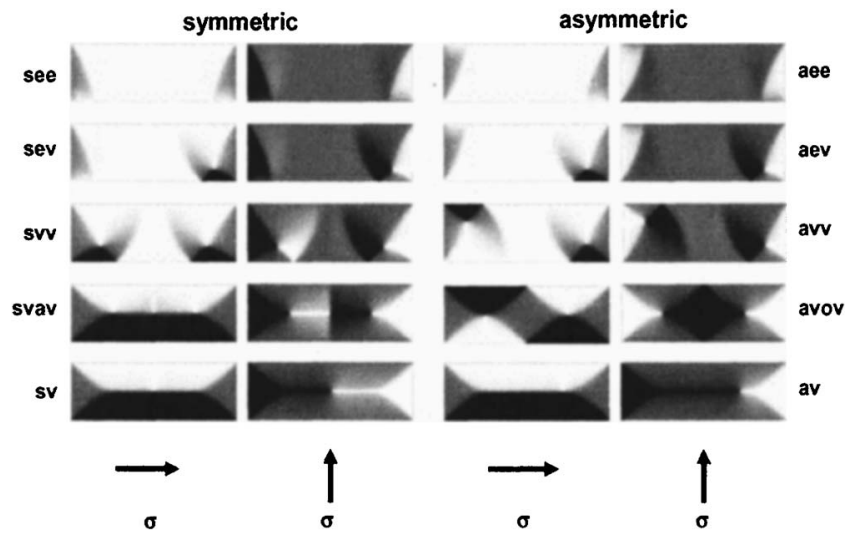

FIG. 1. Simulations of the rectangular domain patterns of Table I. In the white and dark regions the magnetization is parallel and antiparallel to the projection of the direction of the incident light onto the plane of the element and in the medium gray regions it is perpendicular to it as indicated by the arrows. Two illumination directions are shown for each configuration. On the left side are the symmetric configurations and on the right the antisymmetric/asymmetric configurations. For nomenclature see text.

point of view. The influence of magnetocrystalline anisotropy is averaged to zero over any small region that contains a few grains. For the simulation, anisotropy is neglected as an approximation in the absence of a detailed knowledge about grain size and distribution.

Figure 1 shows the simulations of the ten rectangular domain configurations considered. They are plotted and arranged according to their symmetry and to the number of vortices. Symmetric patterns $(s)$ are shown on the left and antisymmetric/asymmetric patterns $(a)$ at the right side of the panel. From top to bottom, the number of vortices increases, starting from the pure edge (quasi)domains $(e)$ via mixed edge-vortex structures $(e v)$ to pure vortex structures $(v)$. For each pattern two orthogonal $\boldsymbol{\sigma}$ directions are shown, with the gray level indicating the $\mathbf{M}$ direction with respect to $\boldsymbol{\sigma}$, ranging from parallel (white) via orthogonal (medium gray) to antiparallel (black). They are from top to bottom: $\mathrm{C}$ pattern (see, left) and S pattern (aee, right), $\rho$ pattern (sev, left) and $\sigma$ pattern (aev, right), $\Omega$ pattern ( $s v v$, left) and $\Sigma$ pattern ( $a v v$, right), cross-tie pattern (svav, left) and diamond pattern (avov, right), and vortex pattern ( $s v$, left) and shifted vortex

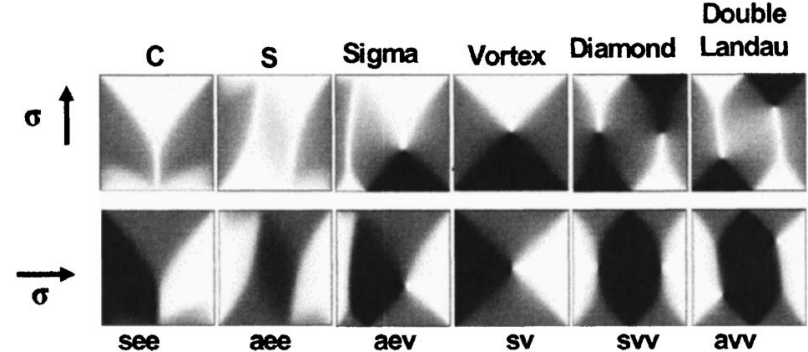

FIG. 2. Simulated magnetization distributions in square elements. All configurations are stable except the diamond pattern that converts into the double Landau patterns during energy minimization driven by the repulsion between the vortices.

pattern ( $a v$, right). In (svav) $a$ means antivortex in the center and in (avov) o means no vortex in the center. Several of them have been simulated and named before in permalloy simulations (Refs. 2 and 4 and references therein). Figure 2 shows the corresponding domain structures in square elements.

The magnetic energy densities of the configurations for the three aspect ratios and element widths are listed in Table I in units of $10^{-4} \mathrm{eV} / \mathrm{nm}^{3}$. The states with the lowest energy are printed in bold. Several tendencies are obvious: (i) the energy densities decrease with increasing aspect ratio, (ii) the energy differences for a given aspect ratio increase with increasing element size, and (iii) the flux-closure patterns such as the diamond and vortex patterns that minimize the stray field energy are in general preferred except in the smallest elements at the largest aspect ratio. In these the (quasi)monodomain states $C$ and $S$ have the lowest total energy because of the large contribution of the exchange energy connected with domain walls. At the aspect ratios 1:1 and 2:1 initial $\Sigma$ patterns convert into the diamond pattern during the energy minimization process and at the aspect ratio 1:1 initial $\rho$, cross-tie, and $\Omega$ patterns convert into the vortex pattern because no significant barrier has to be overcome.

\section{EXPERIMENTAL RESULTS}

Before presenting some XMCDPEEM images representative of the large number of images taken in order to obtain

TABLE I. Total magnetic energy densities of 20-nm-thick rectangular Co elements with aspect ratios of 1:1, 2:1, and 3:1 and widths of 200, 400, and 600 nm. The lowest energies are printed in bold. The energies are in units of $10^{-4} \mathrm{eV} / \mathrm{nm}^{3}$. Where numbers are missing, the original configuration was unstable and converted into a lower-energy configuration. At the aspect ratio 1:1 the diamond pattern converts into the double Landau pattern.

\begin{tabular}{|c|c|c|c|c|c|c|c|c|c|c|}
\hline \multirow[b]{2}{*}{ Symbol } & \multirow[b]{2}{*}{ Name } & \multicolumn{3}{|c|}{$1: 1$} & \multicolumn{3}{|c|}{$1: 2$} & \multicolumn{3}{|c|}{$1: 3$} \\
\hline & & $200 \mathrm{~nm}$ & $400 \mathrm{~nm}$ & $600 \mathrm{~nm}$ & $200 \mathrm{~nm}$ & $400 \mathrm{~nm}$ & $600 \mathrm{~nm}$ & $200 \mathrm{~nm}$ & $400 \mathrm{~nm}$ & $600 \mathrm{~nm}$ \\
\hline see & $C$ & 5.060 & 2.621 & 1.754 & 3.047 & 1.597 & 1.082 & 2.105 & 1.107 & 0.749 \\
\hline aee & $S$ & 5.448 & 2.825 & 1.892 & 3.050 & 1.598 & 1.082 & 2.105 & 1.106 & 0.749 \\
\hline sev & rho & $\cdots$ & $\cdots$ & $\cdots$ & 3.262 & 1.513 & 1.005 & 2.286 & 1.083 & 0.708 \\
\hline aev & sigma & 5.602 & 2.495 & 1.544 & 3.198 & 1.452 & 1.004 & 2.285 & 1.082 & 0.704 \\
\hline svv & Omega & $\cdots$ & $\cdots$ & $\cdots$ & 2.657 & 1.380 & 0.848 & 2.456 & 1.051 & 0.640 \\
\hline avv & Sigma & $\cdots$ & $\cdots$ & $\cdots$ & $\cdots$ & $\cdots$ & $\cdots$ & 2.456 & 1.049 & 0.646 \\
\hline svvv & Crosstie & $\cdots$ & $\cdots$ & $\cdots$ & 2.657 & 1.003 & 0.576 & 2.574 & 1.035 & 0.610 \\
\hline avov & Diamond & dL5.771 & dL2.354 & dL1.388 & 2.497 & 0.929 & 0.526 & 2.179 & 0.858 & 0.506 \\
\hline sv & Vortex & 2.339 & 0.900 & 0.516 & 2.260 & 0.946 & 0.573 & 2.544 & 1.097 & 0.669 \\
\hline av & Shifted vortex & & & & $\cdots$ & $\cdots$ & 0.580 & $\cdots$ & 1.103 & 0.675 \\
\hline
\end{tabular}




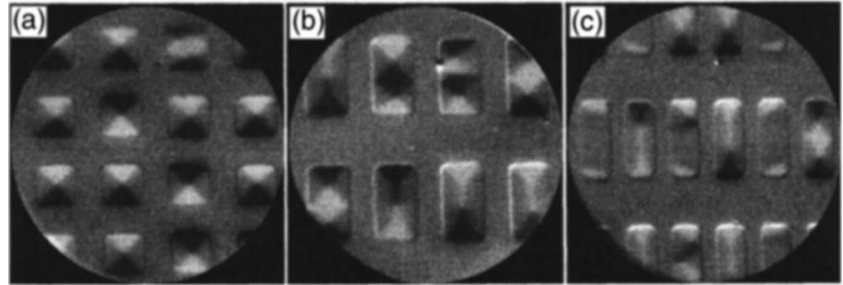

FIG. 3. Representative XMCDPEEM images of 20-nm-thick Co elements with the aspect ratios 1:1 (a, 600×600 nm $), 1: 2\left(\mathrm{~b}, 1200 \times 600 \mathrm{~nm}^{2}\right)$, and 1:3 (c, $1200 \times 400 \mathrm{~nm}^{2}$ ). Diameter of field of view in all cases: $5 \mu \mathrm{m}$. The light is incident in the horizontal direction in all images.

a statistical distribution a few comments are necessary regarding resolution and contrast. As mentioned in Sec. II, these images are the result of the subtraction of two images taken with light of opposite helicity. Typical image acquisition times range from 20 to $300 \mathrm{~s}$ for a field of view of 10and $2.5-\mu \mathrm{m}$ diameters, respectively. Switching the helicity by moving the undulator magnets requires $90 \mathrm{~s}$. During these periods small image shifts may occur that limit resolution. We overcome these limitations largely by collecting a sequence of images (typically 30 frames per helicity) and correcting the image/sample drift after calculating the crosscorrelation between the different sequence frames. The drift correction procedure produces the black/white dot in some of the images shown below. This artifact is due to a blind spot of the channel-plate detector. In addition, the finely focused photon beam frequently drifts so that the two images can have different intensity gradients. As a result, the elements with the same magnetization direction may show slightly different gray levels in the difference (XMCD) image.

Figure 3 shows some typical domain distributions. In smaller elements the magnetization distribution can be seen too but not as clearly because of the limitations mentioned above. In the aspect ratio 1:1 [Fig. 3(a)] the vortex structure is dominating; only in one element in the upper right corner a double Landau pattern is seen. Figure 3(b) (aspect ratio 1:2) shows several vortices, two diamond and one Omega pattern and Fig. 3(c) (aspect ratio 1:3) two $S$, one sigma, one

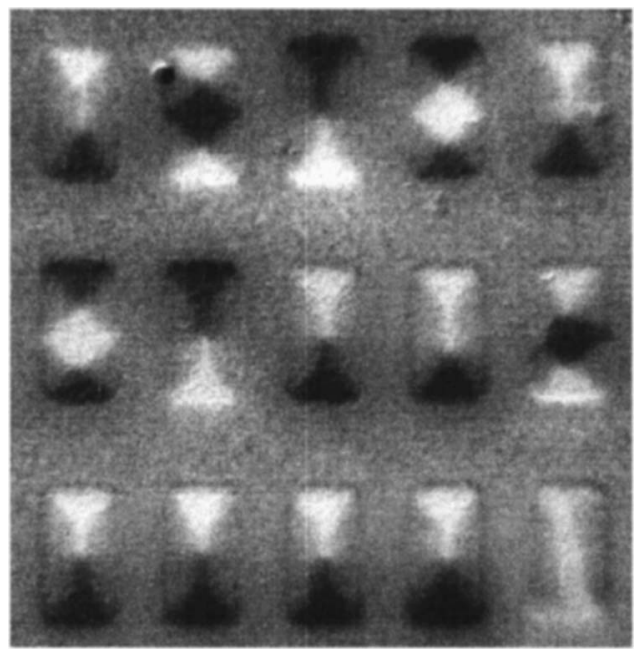

FIG. 4. XMCDPEEM image of 20-nm-thick Co elements with aspect ratio $2: 1\left(1200 \times 600 \mathrm{~nm}^{2}\right)$ showing an unusual pattern in the lower right corner. Image size: $5.2 \times 5.8 \mu \mathrm{m}^{2}$.
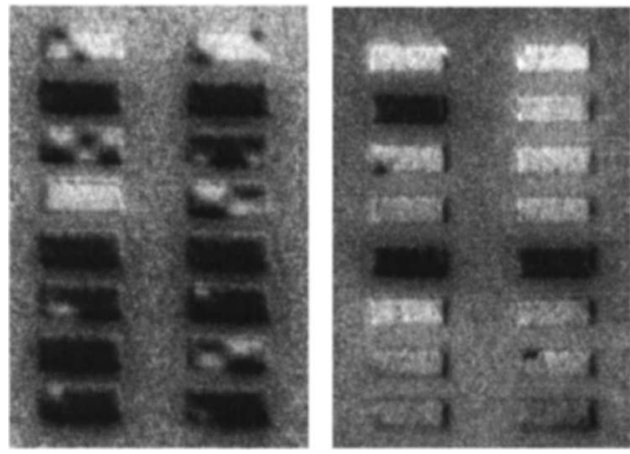

FIG. 5. XMCDPEEM images of the virgin state (left) and of the remanent state (right) of 20-nm-thick Co elements with aspect ratio 2:1 (800 $\times 400 \mathrm{~nm}^{2}$ ). Three of the (quasi)monodomain elements were not switched by the 350 -Oe field applied. Image size: $2.9 \times 4.3 \mu \mathrm{m}^{2}$.

Omega, three diamond and four shifted vortex patterns, and several not fully identifiable edge domain patterns. The relative frequency of the various patterns changes with the size of the elements as will be shown in Sec. V in the comparison with the calculated energies. Occasionally we observed patterns different from all of the simulations presented in Fig. 1. An example is shown in Fig. 4. The element in the lower right corner has horizontal magnetization in the center and regions with vertical magnetization on the sides. In the simulations this configuration is unstable.

It is interesting to point out the difference between virgin and remanent states which is illustrated in Fig. 5. In the virgin state several domain structures are seen, including some (quasi)monodomain states but after exposure to a field of about 350 Oe only (quasi)monodomain states remain, interestingly some with magnetization opposite to the applied field which was obviously not strong enough to switch some of the original (quasi)monodomain elements.

LEEM images were taken from many elements. One of them is shown in Fig. 6. Though possibly not visible in print, there are many small dark and bright spots in the bright elements, the largest of which have about 20-nm diameter. The contrast is caused by electron diffraction because it

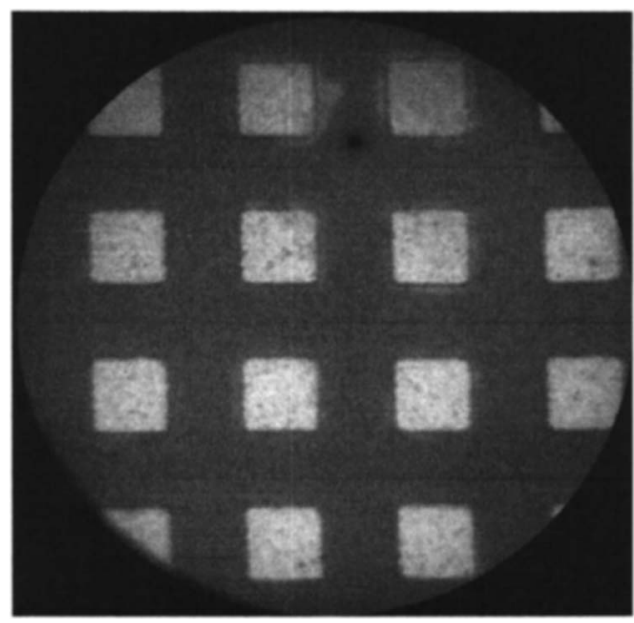

FIG. 6. Typical LEEM image of 20-nm-thick Co elements. The brightness of the elements indicates a preferred orientation of the crystallites with their surface approximately parallel to the film surface. Electron energy: $5.1 \mathrm{eV}$. Diameter of field of view: $10 \mu \mathrm{m}$. 

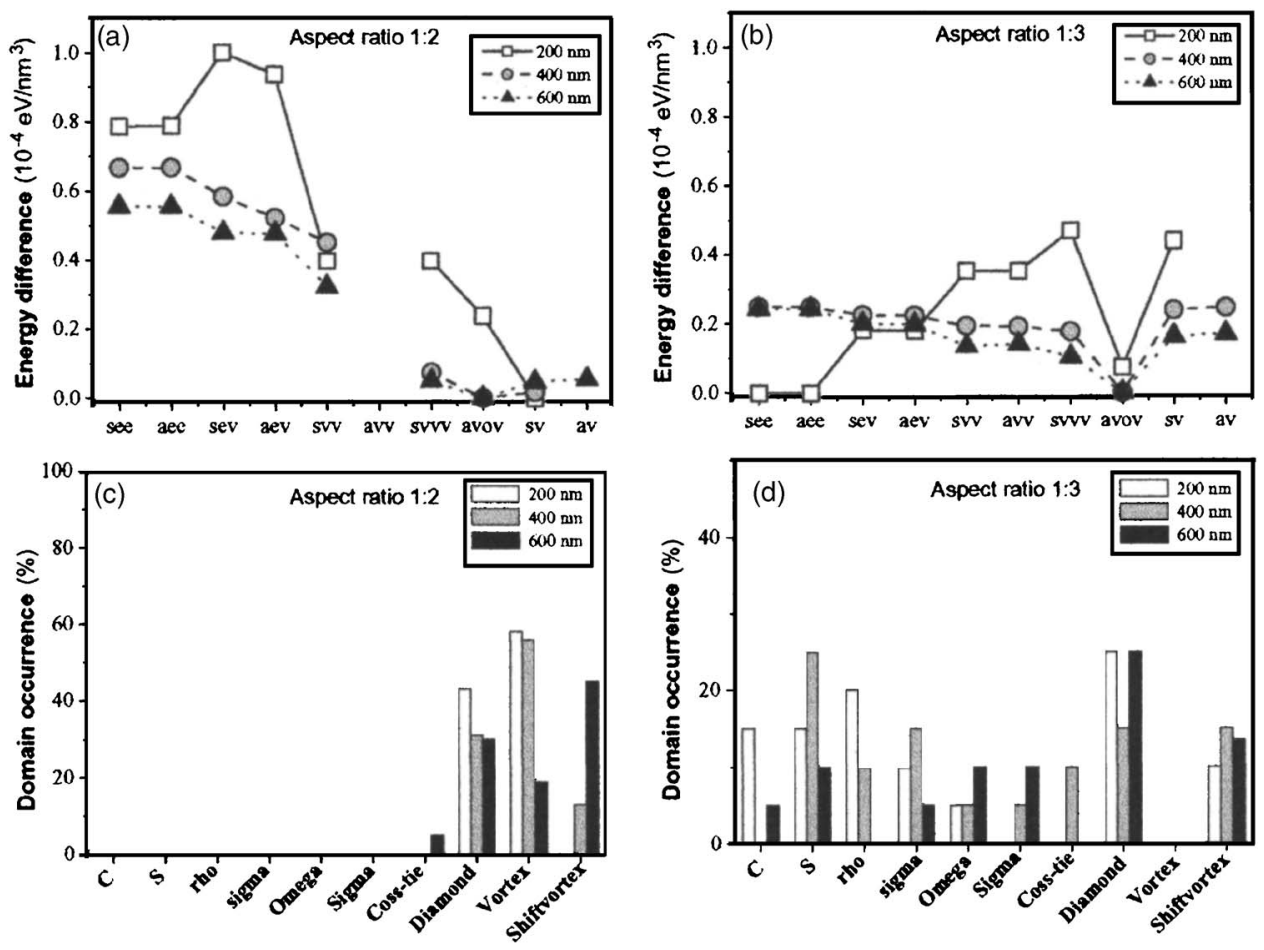

FIG. 7. Top: Energy difference between the various patterns and the pattern with the lowest energy of 20-nm-thick rectangular Co elements with aspect ratios 1:2 (a) and 1:3 (b) in units of $10-4 \mathrm{eV} / \mathrm{nm}^{3}$. Bottom: Corresponding percentages of the various patterns observed by XMCDPEEM (c and d). In (c) all 200-nm-wide vortex patterns are displayed in the vortex column because symmetric and asymmetric vortices cannot be reliably distinguished. changes with electron energy so that the spots represent small crystallites. The brightness of the elements is due to a strong (00) spot in the LEED pattern, probably from (0001) planes, which indicates a preferred orientation of the crystallites. The MEM images that are more sensitive to surface roughness and potential variations support these conclusions.

\section{COMPARISON OF SIMULATION AND EXPERIMENT}

The comparison of simulation and experiment is based on the assumption that the relative probability of the various magnetization configurations is determined by their energy (density) difference. In Fig. 7 we plot the energy density differences between the various simulated configurations and the configuration with the lowest energy ( $a$ and b) and for comparison the relative number of observed configurations (c and d) for the aspect ratios 1:2 (a and c) and 1:3 (b and d). For the aspect ratio 1:1 no plot is needed because the energy differences are so large that only configurations with the lowest energy are statistically expected. In fact, the vortex state was observed in $100 \%, 96 \%$, and $94 \%$ of the $200-, 400-$, and $600-\mathrm{nm}$ wide elements, respectively. $3 \%$ and $6 \%$ of the elements were in the double Landau state in the 400- and 600-nm elements, respectively, and one of the 400-nm elements was unexpectedly in the high- energy $S$ state (see Table I). There is a general agreement insofar as configurations with large energy difference are seldom or never observed and that the number of configurations increases with decreasing energy difference as a comparison of Figs. 7(a) and 7(c) with Figs. 7(b) and 7(d).

There are, however, notable exceptions. For example, the $C, S$, and diamond states are not as dominating in the 200-nm wide elements with aspect ratio 1:3 as one would expect on the basis of the energy differences. Other unexpected results-in view of their higher energies-are the high percentage of $S$ states in the 400-nm wide elements with $1: 3$ aspect ratio and the high percentage of rho states in the 200-nm wide elements with aspect ratio 1:3. Thus, while there is good qualitative agreement between the energy differences of the various domain patterns and their observed relative number, there is no quantitative agreement.

\section{DISCUSSION}

In order to understand the lack of quantitative agreement between the simulation and the experiment one has to keep in mind the differing conditions in the two cases. The simulations find the local energy minimum in the magnetic configuration space nearest to the selected initial configuration. A transition to a local minimum with lower energy requires surmounting an energy barrier which is very unlikely at room temperature, except for very low barriers $\left(E_{\text {act }}\right.$ $\cong k T \approx 0.0255 \mathrm{eV})$. Thus, the initial state-dependent minimum-energy configurations are frozen in.

The initial state in the experiment is quite different. Before lift-off, the patterns are part of a more or less continuous film with magnetic domains that are large compared with those in the patterns observed after lift-off. "More or less" means that the patterns are at a somewhat lower level than the surrounding film which is on top of the photoresist. This should, however, cause only a minor disturbance in the domain structure of the film before lift-off. During lift-off, the field surrounding the pattern is suddenly removed and the pattern acquires one of the domain configurations with local minimum energy.

Thus the initial conditions in the simulation and the experiment are quite different and it is not surprising that there is no quantitative agreement. However, all the low-energy configurations are observed. The high-energy configurations seen occasionally may be attributed to pinning by defects. 
The same cause is probably responsible for the incomplete switching in a magnetic field illustrated in Fig. 5.

The somewhat undefined initial conditions in patterns produced by electron-beam lithography and lift-off or ionbeam milling of continuous films suggest to study individual patterns that are free from these limitations. The simulations for permalloy elements with aspect ratio 1:2 (Ref. 4) show that the state with the lowest total energy changes with increasing thickness from the $C$ or $S$ state, which differ hardly in energy, via the vortex state to the diamond state, for example, in a 500-nm-long element at 7.5 and $26 \mathrm{~nm}$, respectively. Whether or not these transitions can occur during growth depends on the unknown potential barriers between these states.

In view of the fact that the magnetization distribution in small elements can develop during growth it would be highly desirable to study its evolution with increasing thickness. Real time studies of the evolution of the magnetization pattern during growth of one and the same elements have been made for small Co crystals on a W(110) surface (Ref. 9) with spin-polarized low-energy electron microscopy [SPLEEM (Ref. 10)] but the crystals had a large aspect ratio and showed only monodomain behavior. Small crystals with smaller aspect ratio have been grown in the case of $\mathrm{Fe}$ on $\mathrm{Mo}(110)$ and $\mathrm{W}(110)$ and studied with magnetic force microscopy $^{11}$ and spin-polarized scanning tunneling microscopy ${ }^{12,13}$ both in the single domain and multidomain regions. The dependence of the domain structure upon thickness could, however, be demonstrated only on different crystals. In principle the evolution of the domain structure in these crystals could be studied in situ by XMCDPEEM but the slow image acquisition time, high specimen load, and possibly specimen contamination makes this unattractive. SPLEEM is much better suited for this purpose. Of course, the very early states of the evolution of the magnetization from the superparamagnetic limit are below the resolution limit of both techniques and require spin-polarized scanning tunneling microscopy. ${ }^{14}$ For the moment the evolution of the magnetization distribution in small elements with increasing thickness remains an open question.

\section{SUMMARY}

We have studied the magnetic domain structures in small 20-nm-thick rectangular Co elements both theoretically with simulations and experimentally with XMCDPEEM and compared the results of both methods. We have found that qualitatively the relative probability of the various domain patterns is determined by the total energy of the elements but that there are quantitative differences between simulations and experiment that can be attributed to the differing initial states and to potential barriers that cannot be overcome by thermal activation. Future SPLEEM studies will hopefully shed some light onto the evolution of the magnetic domain structure of small elements with increasing thickness.

\section{ACKNOWLEDGMENTS}

This work was supported by the National Science Foundation under Grant No. 9818296 and by the Office of Naval Research Grant No. N-000140210922, monitored by Chagaan Bataar ONR and by NATO.

\footnotetext{
${ }^{1}$ A. Hubert and R. Schaefer, Magnetic Domains (Springer, Berlin, 1998).

${ }^{2}$ W. Rave and A. Hubert, IEEE Trans. Magn. 36, 3886 (2000).

${ }^{3}$ R. Hertel, J. Appl. Phys. 90, 5752 (2001).

${ }^{4}$ R. Hertel, Z. Metallkd. 93, 957 (2002).

${ }^{5}$ J. Stoehr, Y. Wu, B. D. Hermsmeier, M. G. Samant, G. P. Harp, S. Koranda, D. Dunham, and B. P. Tonner, Science 259, 658 (1993).

${ }^{6}$ A. Locatelli, S. Cherifi, S. Heun, M. Marsi, K. Ono, A. Pavlovska, and E. Bauer, Surf. Rev. Lett. 9, 171 (2002).

${ }^{7}$ ELMITEC-GmbH, mail@elmitec.de

${ }^{8}$ A. Locatelli, S. Cherifi, S. Heun, M. Marski, K. Ono, A. Pavlovska, and E. Bauer, J. Phys. I 104, 99 (2003).

${ }^{9}$ H. Pinkvos, H. Poppa, E. Bauer, and G.-M. Kim, in Magnetism and Structure in Systems with Reduced Dimension, edited by R. F. C. Farrow, B. Dierry, M. Donath, A. Fert, and B. D. Hermsmeier (Plenum, New York, 1993), p. 25.

${ }^{10} \mathrm{E}$. Bauer, in Magnetic Microscopy of Nanostructures, edited by H. Hopster and H. P. Oepen (Springer, Berlin, 2004), p. 111.

${ }^{11}$ P. O. Jubert, J. C. Toussaint, O. Fruchart, C. Meger, and Y. Samson, Europhys. Lett. 63, 132 (2003).

${ }^{12}$ A. Yamasaki, W. Wulfhekel, R. Hertel, S. Suga, and J. Kirschner, Phys. Rev. Lett. 91, 127201 (2003).

${ }^{13}$ M. Bode, A. Wachowiak, J. Wiebe, A. Kubetaka, M. Morgernstern, and R. Wiesendanger, Appl. Phys. Lett. 84, 948 (2004).

${ }^{14}$ M. Bode, Rep. Prog. Phys. 66, 206523 (2003).
} 